\title{
Optimal adjuvant chemotherapy completion time for stage III colon cancer: a cohort study
}

\author{
Jing-Qing Ren^, Han-Shuo Zhang, Li-Hua Zhang, Qi-Guang Zhong, Fan Wu, Bai-Lin Wang, Shao-Jie Liu \\ Department of General Surgery, Medical College, Jinan University, Guangzhou Red Cross Hospital, Guangzhou, China \\ Contributions: (I) Conception and design: JQ Ren; (II) Administrative support: SJ Liu; (III) Provision of study materials or patients: HS Zhang, \\ LH Zhang; (IV) Collection and assembly of data: QG Zhong, F Wu; (V) Data analysis and interpretation: BL Wang; (VI) Manuscript writing: All \\ authors; (VII) Final approval of manuscript: All authors. \\ Correspondence to: Shao-Jie Liu. Department of General Surgery Medical College, Jinan University, Guangzhou Red Cross Hospital, 396 Tongfu \\ Middle Rd., Haizhu District, Guangzhou 510220, China. Email: 51242743@qq.com.
}

Background: Adjuvant chemotherapy for 6 months following surgery is the standard treatment plan for stage III colon cancer. The aim of the present study was to determine whether the adjuvant chemotherapy completion time for stage III colon cancer had an effect on prognosis and cut-off time that affected the prognosis.

Methods: This was a retrospective study of stage III colon cancer patients who completed adjuvant chemotherapy at Guangzhou Red Cross Hospital from January 2010 to December 2017. Univariate and multivariate analyses were used to determine the association between adjuvant chemotherapy completion time and the 3-year disease-free survival (DFS). The restricted cubic spline model was used to analyze the cut-off time that affected the 3 -year DFS.

Results: A total of 431 patients were included in the study. The 3-year DFS was associated with a combination of obstruction or perforation, preoperative serum carcino-embryonic antigen (CEA) concentration, $\mathrm{T}$ stage, $\mathrm{N}$ stage, pathological stage, and adjuvant chemotherapy completion time in the univariate analysis $(\mathrm{P}<0.05)$. A combination of obstruction or perforation, preoperative serum CEA concentration, $\mathrm{N}$ stage, and adjuvant chemotherapy completion time were independent prognostic factors in the multivariate analysis $(\mathrm{P}<0.05)$. The cut-off time was 28 weeks for adjuvant chemotherapy completion time in the restricted cubic spline model analysis. For those whose adjuvant chemotherapy completion time was $>28$ weeks, the risk of 3-year recurrence was 1.428 times higher compared with those whose adjuvant chemotherapy completion time was $\leq 28$ weeks. [ $\mathrm{P}=0.032,95 \%$ confidence interval (CI): 1.034-2.055].

Conclusions: The 3-year DFS of stage III colon cancer was related to the adjuvant chemotherapy completion time. For those who completed adjuvant chemotherapy $>28$ weeks, the risk of 3 -year recurrence increased.

Keywords: Colon cancer; adjuvant chemotherapy; completion time; disease-free survival (DFS)

Submitted May 21, 2021. Accepted for publication Jul 06, 2021.

doi: 10.21037/jgo-21-317

View this article at: https://dx.doi.org/10.21037/jgo-21-317

\footnotetext{
^ ORCID: 0000-0001-8426-5337.
} 


\section{Introduction}

There are currently 104,270 new colon cancer cases in the USA every year (1), of which approximately onethird are stage III (2). Even with radical surgery, the postoperative recurrence rate with stage III colon cancer is still as high as $50-80 \%$ (3). Previously published studies have found that adjuvant chemotherapy could reduce the risk of recurrence and improve survival for patients stage III colon cancer (4). Therefore, adjuvant chemotherapy is routinely recommended for stage III colon cancer for 6 months following surgery by the National Comprehensive Cancer Network (5). Based on the results of the Adjuvant Treatment of Colon

Cancer (MOSAIC) (6), oxaliplatin plus fluorouracil and leucovorin (FOLFOX) is the standard adjuvant chemotherapy regimen (7). However, the efficacy of adjuvant chemotherapy has not been found to improve by adding targeted drugs (8). Because modified FOLFOX6 (mFOLFOX6) is easier to manage than FOLFOX4, it has replaced the FOLFOX4 regimen for adjuvant chemotherapy for stage III colon cancer (9). Oral capecitabine combined with oxaliplatin has also demonstrated the same efficacy as mFOLFOX6 (10). Although adjuvant chemotherapy for 6 months following surgery is the standard treatment plan for stage III colon cancer, many patients are unable to complete adjuvant chemotherapy within this timeframe. At present, most studies mainly focus on patients' adjuvant chemotherapy regimens and chemotherapy cycles, but do not pay too much attention to the adjuvant chemotherapy completion time. To date, there is still no guideline to specify the length of time within which patients should complete adjuvant chemotherapy. The aim of the present study was to determine whether the adjuvant chemotherapy completion time for stage III colon cancer has an effect on prognosis and cut-off time that affected the prognosis. We present the following article in accordance with the STROBE reporting checklist (available at https://dx.doi. org/10.21037/jgo-21-317).

\section{Methods}

\section{Patients}

This was a retrospective cohort study. The study was performed in accordance with the Declaration of Helsinki (as revised in 2013). Ethics approval was waived by the Ethics Committee of the Jinan University, Guangzhou Red Cross Hospital (No. AF/SC-107/02.0).
Individual consent of participation in this study for this retrospective analysis was waived. Patients with stage III colon cancer who completed all adjuvant chemotherapy at Guangzhou Red Cross Hospital (Guangzhou, China) from January 2010 to December 2017 were included in the present study. Inclusion criteria were as follows: (I) patients aged 18-70 years; (II) the first adjuvant chemotherapy was 4-8 weeks following surgery; (III) Eastern Cooperative Oncology Group (ECOG) score of $0-1$; and (IV) completion of the adjuvant chemotherapy program [oxaliplatin plus xeloda (XELOX) regimen for 8 cycles and FOLFOX regimen for 12 cycles]. Exclusion criteria were as follows: (I) multiple colon cancer; (II) other previous or concurrent malignant diseases; (III) preoperative neoadjuvant chemotherapy; (IV) recurrence during adjuvant chemotherapy; (V) lost to follow up. Lost to follow up was defined as follow up $<36$ months following surgery with no recurrence at the last follow up. Six cases were lost to follow up (6/437, 1.37\%). The number of cases during the study period determined the sample size. In order to reduce the selection bias, we chose cases with a not long time span (8 years), they had similar chemotherapy initiation time (within 8 weeks after surgery) and similar chemotherapy regimens (FOLFOX or XELOX). A total of 431 cases were included in the present study (Figure 1). All cases were confirmed by pathology. There were 207 males and 224 females, aged from 18 to 70 years (median age: 58 years); 212 cases had right colon cancer (cecum to transverse colon) and 219 had left colon cancer (splenic flexure colon to sigmoid colon) in the study cohort. Fifty-six cases had IIIA stage, 273 cases had IIIB stage, and 102 cases had IIIC stage. The XELOX regimen was assigned for 235 cases and the FOLFOX regimen was assigned for 196 cases as adjuvant chemotherapy.

\section{Chemotherapy}

The patients provided signed informed chemotherapy consent prior to chemotherapy. Adjuvant chemotherapy commenced 4-8 weeks following surgery. The chemotherapy regimen was XELOX (oxaliplatin $130 \mathrm{mg} / \mathrm{m}^{2} \mathrm{~d} 1$ and capecitabine $1,000 \mathrm{mg} / \mathrm{m}^{2} \mathrm{~d} 1-\mathrm{d} 14$, repeated for 3 weeks for a total of 8 cycles) or FOLFOX (oxaliplatin $85 \mathrm{mg} / \mathrm{m}^{2} \mathrm{~d} 1$; leucovorin $200 \mathrm{mg} / \mathrm{m}^{2} \mathrm{~d} 1$, d2; fluorouracil $400 \mathrm{mg} / \mathrm{m}^{2} \mathrm{~d} 1$, $\mathrm{d} 2$; fluorouracil $600 \mathrm{mg} / \mathrm{m}^{2} \mathrm{~d} 1, \mathrm{~d} 2$ by continuous intravenous infusion for 22 hours; or oxaliplatin $85 \mathrm{mg} / \mathrm{m}^{2} \mathrm{~d} 1$, leucovorin $400 \mathrm{mg} / \mathrm{m}^{2} \mathrm{~d} 1$, fluorouracil $400 \mathrm{mg} / \mathrm{m}^{2} \mathrm{~d} 1$, and fluorouracil 


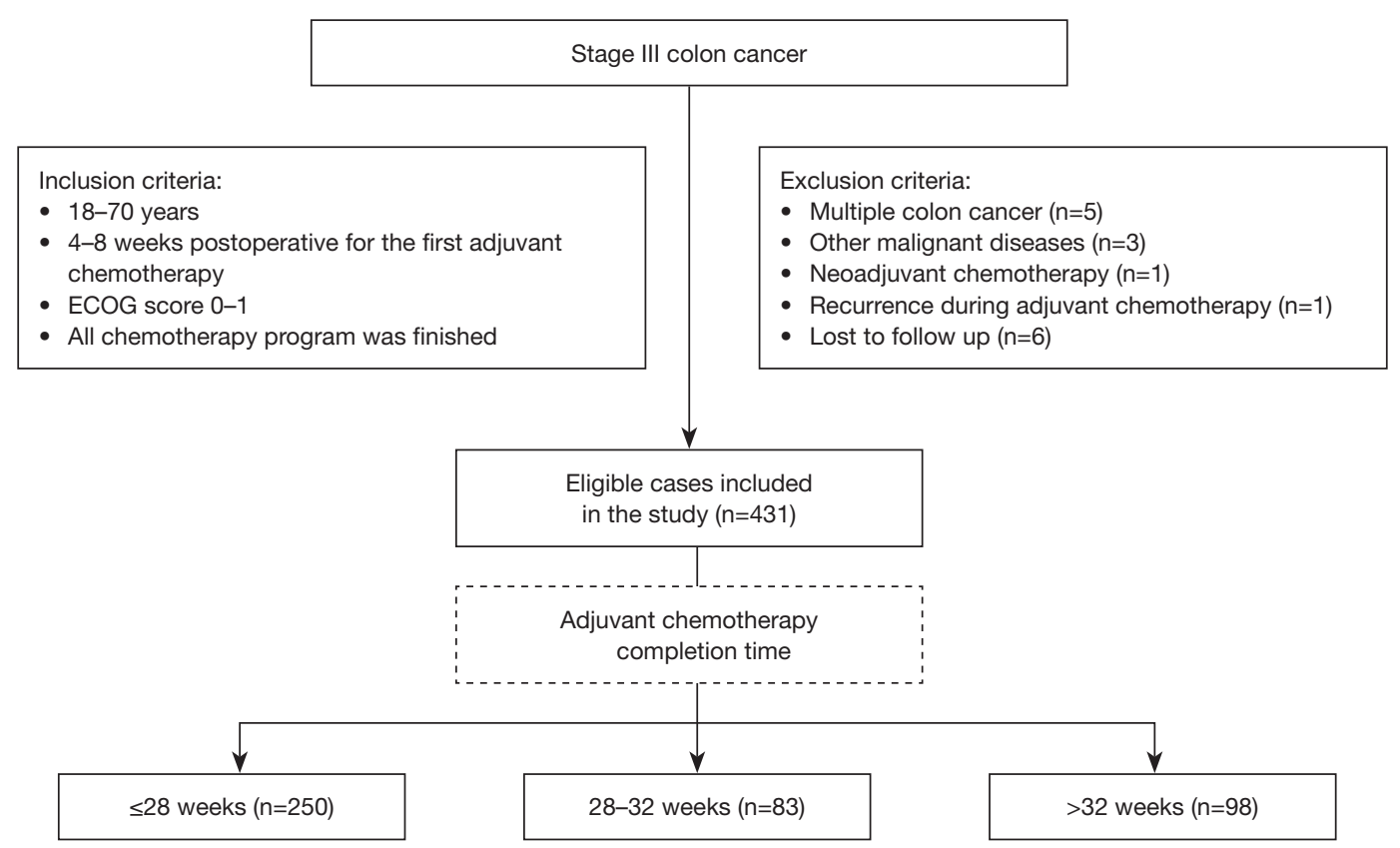

Figure 1 Inclusion of cases in the study. ECOG, Eastern Cooperative Oncology Group.

$2,400 \mathrm{mg} / \mathrm{m}^{2}$ by continuous intravenous infusion for 46 hours, repeated for 2 weeks for a total of 12 cycles). The chemotherapy completion time was calculated from the first chemotherapy to the last chemotherapy (weeks). The chemotherapy completion time was divided into 4-week breakdown points $(\leq 28,28-32$, and $>32$ weeks $)$. This stratification was based on the distribution of the index, so as to guarantee easy interpretation and a sufficient observation with each category.

\section{Follow up}

Patients were followed up every 3 months for the first 2 years after surgery, every 6 months for 2-5 years, and once per year after 5 years. The follow up included detailed medical history, physical examination, serological examination [blood routine, biochemical, carcinoembryonic antigen (CEA), carbohydrate antigen 19-9 (CA19-9)], imaging examinations [e.g., X-ray, B-ultrasound, computed tomography (CT), magnetic resonance imaging (MRI), positron emission tomography (PET)/CT] and colonoscopy. Recurrence was confirmed by CT, MRI, PET/ CT, or pathology. In the event of a discrepancy for imaging examinations, confirmation was made with pathology. The deadline for follow up was December 2020, and the median follow-up time was 43.6 months (8.2-103.3 months). There were no missing data in the study.

\section{Statistical analysis}

SPSS version 23.0 (SPSS, Armonk, NY, USA) and R software (R-4.0.4, www.r-project.org) were used in the study. Count data were expressed as percentages, and continuous variables were expressed as $\bar{x} \pm$ s. Disease-free survival (DFS) was calculated by Kaplan-Meier method, and differences between groups were tested by log-rank test. The independent risk factors for 3-year DFS were analyzed by the Cox proportional hazard model. All cases underwent subgroup analysis according to the different pathological stages. The restricted cubic spline model was used to analyze the association between the adjuvant chemotherapy completion time and the 3-year DFS by the rcspline.plot function in the rms package of the $\mathrm{R}$ software. The adjuvant chemotherapy completion time was used as variable $\mathrm{X}$, and the follow-up time was used as variable $\mathrm{Y}$. The outcome variable was recurrence within 3 years after surgery. Meaningful multifactor variables were balanced before the curve, and turning points of the curve were determined. Three to five nodes were selected to draw the curve first and the cure combination with smaller the Akaike information criterion (AIC) value was chosen. $\mathrm{P}<0.05$ indicated statistically significant differences. 


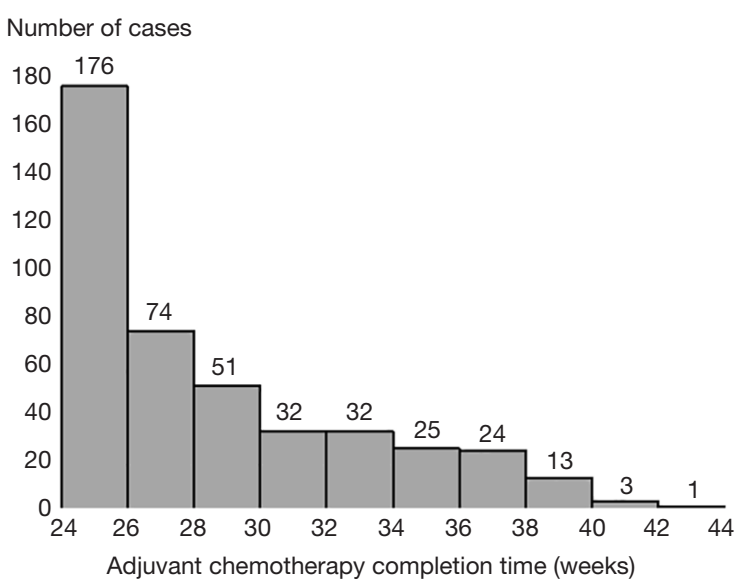

Figure 2 Distribution of adjuvant chemotherapy completion time.

\section{Results}

\section{Adjuvant chemotherapy completion time}

The adjuvant chemotherapy completion time for the entire cohort was 24-43 weeks, with a median of 27 weeks. Of the cohort, the adjuvant chemotherapy completion time was $\leq 28$ weeks for 250 cases (58\%), 28-32 weeks for 83 cases $(19.26 \%)$, and $>32$ weeks for 98 cases $(22.74 \%)$. The distribution of the adjuvant chemotherapy completion time is shown in Figure 2.

\section{Recurrence}

Of the 431 patients, 138 (32.02\%) had recurrence within 3 years after surgery. Of these, 74 (53.62\%) had liver metastasis, 29 (21.01\%) had abdominal metastasis, 26 (18.84\%) had lung metastasis, 14 (10.14\%) had lymph node metastasis, 10 (7.24\%) had bone metastasis, 5 (3.62\%) had ovarian metastasis, 3 had brain metastasis $(2.17 \%)$, $2(1.45 \%)$ had local recurrence, and $3(2.17 \%)$ had other metastases.

\section{Univariate analysis results}

The 3-year DFS was found to be associated with a combination obstruction or perforation, preoperative serum CEA concentration, $\mathrm{T}$ stage, $\mathrm{N}$ stage, pathological stage, and adjuvant chemotherapy completion time in the univariate analysis $(\mathrm{P}<0.05)$. There was no association with sex, age, body mass index (BMI), tumor location, tumor size, tumor configuration, differentiation, and chemotherapy regimen
(Table 1). The DFS for different adjuvant chemotherapy completion times is shown in Figure 3.

\section{Multivariate analysis results}

A combination of obstruction or perforation, preoperative serum CEA concentration, $\mathrm{N}$ stage, and adjuvant chemotherapy completion time were independent prognostic factors for 3-year DFS in the multivariate analysis (Table 2).

\section{Subgroup analysis}

All cases underwent subgroup analysis according to the different pathological stages. It was found that 3 -year DFS decreased with prolonged adjuvant chemotherapy completion time for stage IIIB ( $\mathrm{P}=0.018)$. However, for stage IIIA, no statistical difference was seen for 3-year DFS among different adjuvant chemotherapy completion time groups $(\mathrm{P}=0.890)$. For stage IIIC, the 3 -year DFS tended to decrease with prolonged adjuvant chemotherapy completion time, but no statistical difference was seen, probably due to the small sample size $(\mathrm{P}=0.848)$ (Figures 4-6).

\section{Restrictive cubic spline results}

Restricted cubic spline model analysis was performed on 431 patients. After several factors were balanced, including a combination of obstruction or perforation, preoperative serum CEA concentration, and $\mathrm{N}$ stage, 3 nodes (P5, P50, P95) were selected according to the AIC value. Non-linearity was found between 3-year DFS and the adjuvant chemotherapy completion time (nonlinear test, $\mathrm{P}=0.038$ ). The cut-off time was 28 weeks for adjuvant chemotherapy completion time in the restricted cubic spline model analysis. For those whose adjuvant chemotherapy completion time was $>28$ weeks, the risk of 3 -year recurrence was 1.428 times higher compared with those whose adjuvant chemotherapy completion time was $\leq 28$ weeks $[\mathrm{P}=0.032,95 \%$ confidence interval $(\mathrm{CI}): 1.034$ 2.055] (Figure 7).

\section{Discussion}

Three-year DFS of stage III colon cancer was found to be associated with adjuvant chemotherapy completion time in the present study. The cut-off time was 28 weeks 
Table 1 Univariate analysis of 3-year DFS for stage III colon cancer

\begin{tabular}{|c|c|c|c|}
\hline Variables & No. cases & 3-year DFS (\%) & $P$ value \\
\hline \multicolumn{3}{|l|}{ Sex } & 0.587 \\
\hline Male & 207 & 69.1 & \\
\hline Female & 224 & 67.0 & \\
\hline \multicolumn{3}{|l|}{ Age (years) } & 0.778 \\
\hline$<60$ & 247 & 68.8 & \\
\hline$\geq 60$ & 184 & 66.8 & \\
\hline \multicolumn{3}{|c|}{ Body mass index $\left(\mathrm{kg} / \mathrm{m}^{2}\right)$} & 0.970 \\
\hline$\leq 24$ & 265 & 67.9 & \\
\hline$>24$ & 166 & 68.1 & \\
\hline \multicolumn{3}{|l|}{ Tumor site } & 0.891 \\
\hline Right colon & 212 & 68.4 & \\
\hline Left colon & 219 & 67.6 & \\
\hline \multicolumn{3}{|c|}{ Tumor size (cm) } & 0.546 \\
\hline$\leq 5$ & 236 & 69.1 & \\
\hline$>5$ & 195 & 66.7 & \\
\hline \multicolumn{3}{|c|}{ Tumor configuration } & 0.628 \\
\hline Exophytic & 127 & 71.7 & \\
\hline Ulcerative & 215 & 66.5 & \\
\hline Infiltrative & 89 & 66.3 & \\
\hline \multicolumn{3}{|c|}{ Obstruction or perforation } & 0.006 \\
\hline No & 342 & 70.8 & \\
\hline Yes & 89 & 57.3 & \\
\hline \multicolumn{3}{|l|}{ Differentiation } & 0.348 \\
\hline High & 34 & 73.5 & \\
\hline Moderate & 266 & 68.8 & \\
\hline Poor & 131 & 64.9 & \\
\hline \multicolumn{3}{|c|}{ Serum CEA concentration } & 0.011 \\
\hline Normal & 189 & 74.1 & \\
\hline Abnormal & 242 & 63.2 & \\
\hline \multicolumn{3}{|l|}{ T stage } & 0.023 \\
\hline $\mathrm{T} 1 / \mathrm{T} 2$ & 72 & 79.2 & \\
\hline T3 & 200 & 69.5 & \\
\hline $\mathrm{T} 4$ & 159 & 61.0 & \\
\hline
\end{tabular}

Table 1 (continued)
Table 1 (continued)

\begin{tabular}{lccc}
\hline Variables & No. cases & 3-year DFS (\%) & P value \\
\hline N stage & & & 0.020 \\
N1c & 19 & 84.2 & \\
N1 & 256 & 70.7 & \\
N2a & 93 & 65.6 & \\
N2b & 63 & 55.6 & 0.010 \\
Pathological stage & & & \\
IIIA & 56 & 82.1 & \\
IIIB & 273 & 68.1 & 0.010 \\
IIIC & 102 & 59.8 & \\
Chemotherapy completion time & & \\
(weeks) & & & \\
S28 & 250 & 73.2 & 0.527 \\
28-32 & 83 & 67.5 & \\
>32 & 98 & 55.1 & \\
Chemotherapy regimen & & 68.9 & \\
XELOX & 235 & & \\
FOLFOX & 196 & & \\
\hline
\end{tabular}

DFS, disease-free survival; CEA, carcino-embryonic antigen; FOLFOX, oxaliplatin plus fluorouracil and leucovorin; XELOX, oxaliplatin plus xeloda.

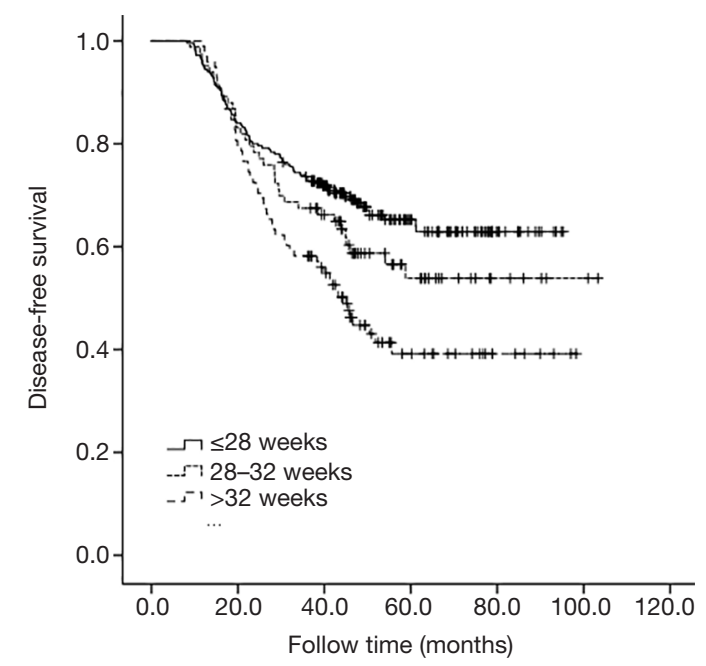

Figure 3 Disease-free survival for different adjuvant chemotherapy completion times. 
Table 2 Multivariate analysis of 3-year disease-free survival for stage III colon cancer

\begin{tabular}{lccccccc}
\hline Variables & $\mathrm{B}$ & Standard error & Wald & $\mathrm{P}$ valve & Exp (B) & Lower 95\% Cl & Upper 95\% Cl \\
\hline Obstruction or perforation & 0.487 & 0.195 & 6.241 & 0.012 & 1.627 & 1.111 & 2.384 \\
Serum CEA concentration & 0.484 & 0.180 & 7.189 & 0.007 & 1.622 & 1.139 & 2.311 \\
T stage & 0.268 & 0.148 & 3.311 & 0.069 & 1.308 & 0.979 & 1.747 \\
N stage & 0.429 & 0.190 & 5.102 & 0.024 & 1.536 & 1.058 & 2.230 \\
Pathological stage & -0.335 & 0.337 & 0.985 & 0.321 & 0.715 & 0.369 & 1.386 \\
Chemotherapy completion time & 0.275 & 0.100 & 7.597 & 0.006 & 1.316 & 1.083 & 1.600 \\
\hline
\end{tabular}

CEA, carcino-embryonic antigen; Cl confidence interval.

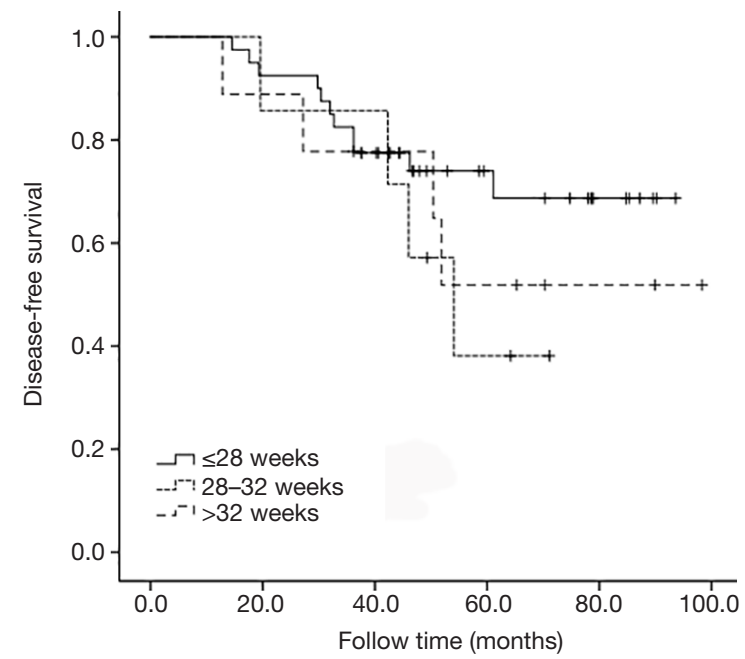

Figure 4 Disease-free survival of stage IIIA colon cancer for different adjuvant chemotherapy completion times.

for those whose adjuvant chemotherapy completion time was $>28$ weeks; however, the risk of 3-year recurrence was 1.428 times higher compared with those whose adjuvant chemotherapy completion time was $\leq 28$ weeks.

In the late 1980s and early 1990s, adjuvant therapy for stage III colon cancer was the standard of care, with adjuvant chemotherapy also constantly being optimized (11). In Dencausse et al.'s study, fluorouracil intravenous bolus chemotherapy for 12 months was used as the initial treatment (12). Then it was found that adjuvant chemotherapy lasting 6 months was not inferior to 12 months in multiple studies (13). In the MOSAIC trial, oxaliplatin combined with fluorouracil for 6-month adjuvant chemotherapy was used the standard treatment plan for stage III colon cancer (6).

A recent study conducted by the International Duration
Evaluation of Adjuvant Chemotherapy collaboration found that, when patients with stage III colon cancer received 3 months of adjuvant chemotherapy, the overall grade 3-4 adverse events and peripheral sensory neuropathy (PSN) were significantly lower than that of patients who received 6 months of adjuvant chemotherapy (14). The DFS in the low-risk group (T1-T3 and N1) was similar regardless of whether they received 3 or 6 months of adjuvant chemotherapy, but the DFS was higher in the high-risk group (T4 and/or N2) when they received 6 months of adjuvant chemotherapy (14). Therefore, 6 months of adjuvant chemotherapy is currently recommended for stage III colon cancer patients at high risk (T4 and/or N2) by American Society of Clinical Oncology (ASCO). For patients at low risk (T1-T3 and N1), 3 or 6 months of adjuvant chemotherapy is recommended, after discussing the potential benefits and risks with patients (15). Prior to 2018 , studies on 3 months of adjuvant chemotherapy studies were rarely conducted. The present study of patients with stage III colon cancer was 2010-2017, and all patients received the planned 6 months of adjuvant chemotherapy.

However, many patients experienced mild-to-severe toxicity during the standard 6-month adjuvant chemotherapy, including PSN, diarrhea, nausea, vomiting, marrow suppression, and fatigue (16). Data from the International Duration Evaluation of Adjuvant Chemotherapy (IDEA) study, half of the patients experienced at least 1 grade 3-4 adverse event, which led to a change or discontinuation in the treatment plan (14). Of these adverse events, oxaliplatininduced cumulative PSN was the most serious, and few patients could recover from PSN after chemotherapy (17). In the MOSAIC, MASCOT, and JOIN trials, grade $>3$ PSN was $12.4 \%, 5.7 \%$, and $5.8 \%$, respectively, which partly led to the low chemotherapy completion rates in the three studies (18-20). In addition to treatment-related 


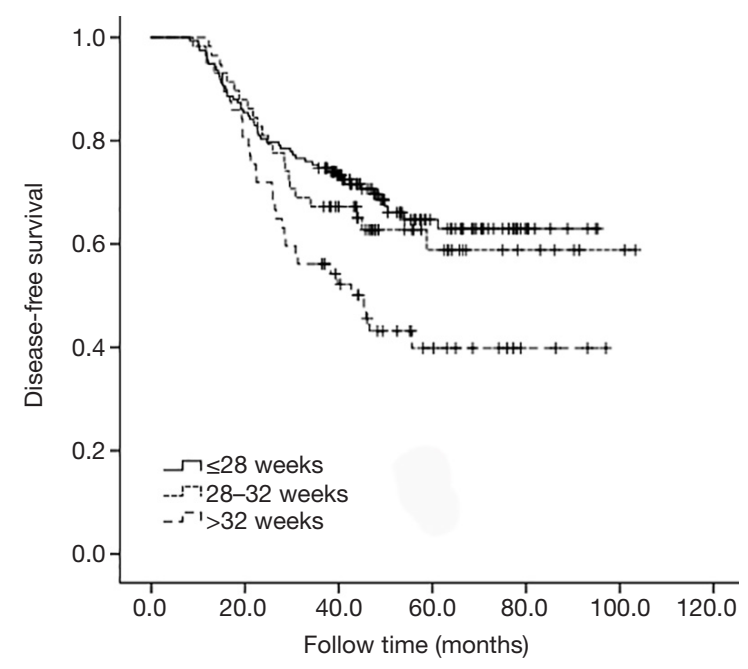

Figure 5 Disease-free survival of stage IIIB colon cancer for different adjuvant chemotherapy completion times.

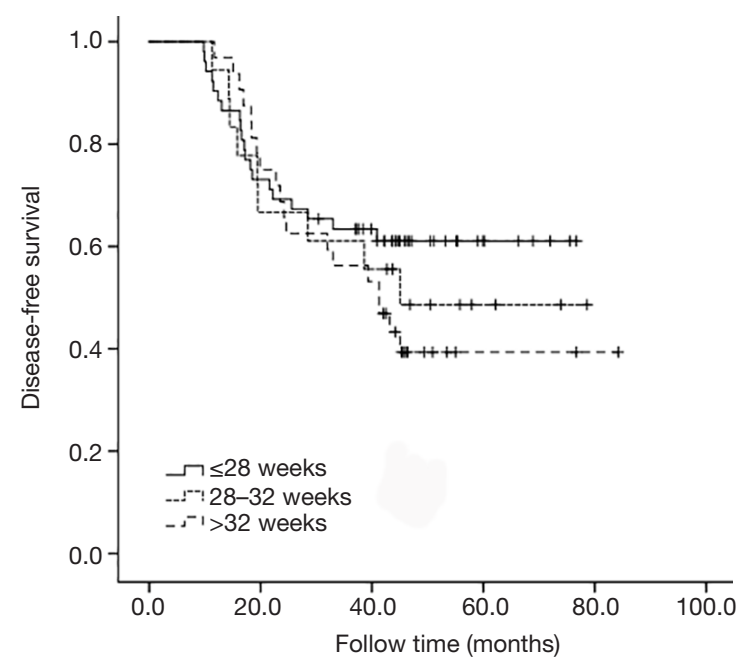

Figure 6 Disease-free survival of stage IIIC colon cancer for different adjuvant chemotherapy completion times.

adverse events, non-medical factors might cause individuals to be reluctant to complete chemotherapy or prolong the completion time, such as treatment costs and access. For patients who had not completed adjuvant chemotherapy, the DFS was significantly lower (21), so it was difficult to evaluate the effect of chemotherapy completion time on prognosis. Therefore, only patients who had completed all chemotherapy plans (XELOX regimen for 8 cycles, and FOLFOX regimen for 12 cycles) were included in the present study.

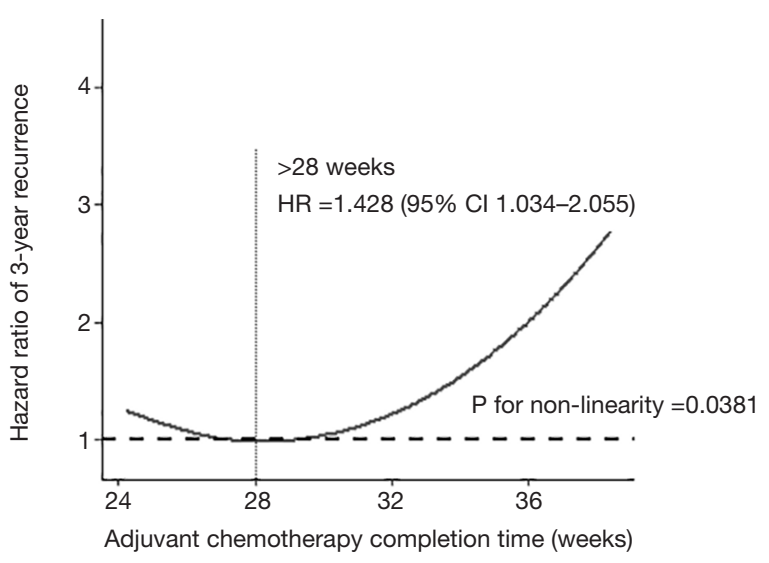

Figure 7 Association between HR of 3-year recurrence and adjuvant chemotherapy completion time. HR, hazard ratio; CI, confidence interval.

Adjuvant chemotherapy generally started within 4-8 weeks following surgery (22). Some scholars believed that the optimal time for stage III colon cancer patients to receive adjuvant chemotherapy was within 8 weeks (23). Delayed chemotherapy led to a higher risk of recurrence and mortality (24). When the initial adjuvant chemotherapy was $>21$ weeks following surgery, the patient could no longer benefit from adjuvant chemotherapy (25). However, few studies have focused on the association between chemotherapy completion time and prognosis. In Jeong et al.'s study, prolonged adjuvant chemotherapy was not related to 5-year DFS (26). The 5-year DFS for the group with $<200$ days of chemotherapy was $77.21 \%$, and $81.82 \%$ for the group with $>200$ days of chemotherapy. However, in that study, up to $28.8 \%$ of stage II cases were included, and the proportion of stage II cases in the 2 groups was not similar; the chemotherapy regimens were also incomparable. In the group with $<200$ days of chemotherapy, $93.8 \%$ of the cases received the fluorouracil plus leucovorin (FL) regimen, but in the group with $>200$ days of chemotherapy, $98.7 \%$ of the cases received the FOLFOX4 regimen. Maybe the conclusion that prolonged adjuvant chemotherapy was not related to 5-year DFS was not convincing. In our study, only patients with stage III colon cancer were included, and adjuvant chemotherapy commenced 4-8 weeks following surgery. The chemotherapy regimen was FOLFOX or XELOX, which had equivalent chemotherapy effects. Therefore, our research results might be more reliable than Jeong et al.'s. We found that prolonged chemotherapy 
completion time could lead to a decrease in DFS. The possible mechanisms were that occult metastases already existed at the time of surgery, and surgery was not a true radical resection (27). This also meant that it would take some time to find the recurrence detected by imaging after the operation. During this period, the tumor burden was the smallest, and theoretically the most susceptible to system therapy. Excessive adjuvant chemotherapy completion time may lead to failure in controlling micrometastatic proliferation in a timely manner (28). Traditional imaging detection methods and tumor markers provided limited information for occult metastases of colorectal cancer. In recent years, circulating tumor cells (CTCs) detection had received increasing attention as a new tumor diagnosis and monitoring method. CTCs detection, which was called "real-time liquid biopsy", was a minimally invasive and convenient way to achieve the purpose of biopsy. It had been confirmed that the dynamic change of its count was related to the efficacy, recurrence, and metastasis of colorectal cancer (29). The detection of CTCs in the peripheral blood circulation indicated that the tumor might have metastasized. Immunosuppressive effects have been confirmed in major surgery (30). Therefore, any unresected subclinical disease would provide good conditions for proliferation following surgery. The reduction in quantity or the prolongation of adjuvant chemotherapy might lead to an increase in recurrence (21). The reduction of angiogenesis inhibitors after tumor resection can lead to an increase in the risk of metastasis (31), and prolonged adjuvant chemotherapy time can make it difficult to inhibit tumor proliferation. Universal superlinear metabolic scaling laws exist in human cancers. This dependence underpins tumor aggressiveness, owing to evolutionary dynamics. As the tumor grows, its proliferation rate may be faster (32). Therefore, the longer the interval between surgery and chemotherapy, the more difficult it is to control the occult metastasis.

This was a retrospective study performed at a single center. We could not know the exact reasons that lead to prolonged adjuvant chemotherapy. Different patients, doctors, or social factors might have an impact on the implementation of the treatment plan. For patients with prolonged adjuvant chemotherapy, the interval between each chemotherapy treatment plan was not similar. And these factors such as patients, doctors, social factors and chemotherapy interval may be associated with postoperative recurrence. In addition, due to the small sample size, it was difficult to make accurate judgments on the cut-off time of adjuvant chemotherapy that affected the prognosis.

Our study discusses the optimization of adjuvant chemotherapy for stage III colon cancer. We found that it was best to complete chemotherapy on schedule, even if it had to be extended, but preferably no more than 28 weeks. Prolonged chemotherapy is associated with colon cancer recurrence. In order to complete chemotherapy on schedule for patients, it is important to address the causes of prolonged chemotherapy.

\section{Conclusions}

The 3-year DFS of patients with stage III colon cancer is associated with the adjuvant chemotherapy completion time. For those who complete $>28$ weeks of adjuvant chemotherapy, the risk of 3 -year recurrence increases.

\section{Acknowledgments}

Funding: None.

\section{Footnote}

Reporting Checklist: The authors have completed the STROBE reporting checklist. Available at https://dx.doi. org/10.21037/jgo-21-317

Data Sharing Statement: Available at https://dx.doi. org/10.21037/jgo-21-317

Conflicts of Interest: All authors have completed the ICMJE uniform disclosure form (available at https://dx.doi. org/10.21037/jgo-21-317). The authors have no conflicts of interest to declare.

Ethical Statement: The authors are accountable for all aspects of the work in ensuring that questions related to the accuracy or integrity of any part of the work are appropriately investigated and resolved. The study was performed in accordance with the Declaration of Helsinki (as revised in 2013). Ethics approval was waived by the Ethics Committee of the Jinan University, Guangzhou Red Cross Hospital (No. AF/SC-107/02.0). The patients provided signed informed chemotherapy consent prior to chemotherapy. Individual consent of participation in this study for this retrospective analysis was waived.

Open Access Statement: This is an Open Access article 
distributed in accordance with the Creative Commons Attribution-NonCommercial-NoDerivs 4.0 International License (CC BY-NC-ND 4.0), which permits the noncommercial replication and distribution of the article with the strict proviso that no changes or edits are made and the original work is properly cited (including links to both the formal publication through the relevant DOI and the license). See: https://creativecommons.org/licenses/by-nc-nd/4.0/.

\section{References}

1. Siegel RL, Miller KD, Fuchs HE, et al. Cancer Statistics, 2021. CA Cancer J Clin 2021;71:7-33.

2. Amin MB, Greene FL, Edge SB, et al. The Eighth Edition AJCC Cancer Staging Manual: Continuing to build a bridge from a population-based to a more "personalized" approach to cancer staging. CA Cancer J Clin 2017;67:93-9.

3. Kanwar SS, Poolla A, Majumdar AP. Regulation of colon cancer recurrence and development of therapeutic strategies. World J Gastrointest Pathophysiol 2012;3:1-9.

4. Taieb J, Gallois C. Adjuvant chemotherapy for stage III colon cancer. Cancers (Basel) 2020;12:2679-86.

5. Provenzale D, Ness RM, Lor X, et al. NCCN guidelines insights: colorectal cancer screening, version 2.2020. J Natl Compr Canc Netw 2020;18:1312-20.

6. André T, Boni C, Mounedji-Boudiaf L, et al. Oxaliplatin, fluorouracil, and leucovorin as adjuvant treatment for colon cancer. N Engl J Med 2004;350:2343-51.

7. Hoehn RS, Smith JJ. Adjuvant chemotherapy for colon cancer. Dis Colon Rectum 2019;62:274-8.

8. André T, Vernerey D, Im SA, et al. Bevacizumab as adjuvant treatment of colon cancer: updated results from the S-AVANT phase III study by the GERCOR Group. Ann Oncol 2020;31:246-56.

9. Kosugi C, Koda K, Ishibashi K, et al. Safety of mFOLFOX6/XELOX as adjuvant chemotherapy after curative resection of stage III colon cancer: phase II clinical study (The FACOS study). Int J Colorectal Dis 2018;33:809-17.

10. Nakayama G, Takano N, Taniguchi H, et al. Randomised phase II trial of capecitabine plus oxaliplatin with continuous versus intermittent use of oxaliplatin as adjuvant chemotherapy for stage II/III colon cancer (CCOG-1302 study). Eur J Cancer 2021;144:61-71.

11. NIH consensus conference. Adjuvant therapy for patients with colon and rectal cancer. JAMA 1990;264:1444-50.

12. Dencausse Y, Hartung G, Sturm J, et al. Adjuvant chemotherapy in stage III colon cancer with 5-fluorouracil and levamisole versus 5 -fluorouracil and leucovorin.

Onkologie 2002;25:426-30.

13. Haller DG, Catalano PJ, Macdonald JS, et al. Phase III study of fluorouracil, leucovorin, and levamisole in highrisk stage II and III colon cancer: final report of intergroup 0089. J Clin Oncol 2005;23:8671-8.

14. Grothey A, Sobrero AF, Shields AF, et al. Duration of adjuvant chemotherapy for stage III colon cancer. N Engl J Med 2018;378:1177-88.

15. Lieu C, Kennedy EB, Bergsland E, et al. Duration of oxaliplatin-containing adjuvant therapy for stage III colon cancer: ASCO clinical practice guideline. J Clin Oncol 2019;37:1436-47.

16. Boyne DJ, O'Sullivan DE, Heer EV, et al. Prognostic factors of adjuvant chemotherapy discontinuation among stage III colon cancer patients: A survey of medical oncologists and a systematic review and meta-analysis. Cancer Med 2020;9:1613-27.

17. Selvy M, Pereira B, Kerckhove N, et al. Long-Term Prevalence of Sensory Chemotherapy-Induced Peripheral Neuropathy for 5 Years after Adjuvant FOLFOX Chemotherapy to Treat Colorectal Cancer: A Multicenter Cross-Sectional Study. J Clin Med 2020;9:2400.

18. André T, Boni C, Navarro M, et al. Improved overall survival with oxaliplatin, fluorouracil, and leucovorin as adjuvant treatment in stage II or III colon cancer in the MOSAIC trial. J Clin Oncol 2009;27:3109-16.

19. Lee PH, Park YS, Ji JF, et al. Safety and tolerability of FOLFOX4 in the adjuvant treatment of colon cancer in Asian patients: The MASCOT study. Asia Pac J Clin Oncol 2009;5:101-10.

20. Kotaka M, Yoshino T, Oba K, et al. Initial safety report on the tolerability of modified FOLFOX6 as adjuvant therapy in patients with curatively resected stage II or III colon cancer (JFMC41-1001-C2: JOIN trial). Cancer Chemother Pharmacol 2015;76:75-84.

21. Yoshimatsu K, Ishibashi K, Koda K, et al. A Japanese multicenter phase II study of adjuvant chemotherapy with mFOLFOX6/CAPOX for stage III colon cancer treatment after D2/D3 lymphadenectomy. Surg Today 2019;49:498-506.

22. Melosky B, Peixoto RD, Karimuddin AA, et al. Reducing time from colon cancer surgery to initiation of adjuvant chemotherapy: pilot project. Can J Surg 2020;63:E223-5.

23. Turner MC, Farrow NE, Rhodin KE, et al. Delay in Adjuvant Chemotherapy and Survival Advantage in Stage III Colon Cancer. J Am Coll Surg 2018;226:670-678. 
24. Becerra AZ, Aquina CT, Mohile SG, et al. Variation in delayed time to adjuvant chemotherapy and diseasespecific survival in stage III colon cancer patients. Ann Surg Oncol 2017;24:1610-7.

25. Gao P, Huang XZ, Song YX, et al. Impact of timing of adjuvant chemotherapy on survival in stage III colon cancer: a population-based study. BMC Cancer 2018;18:234-48.

26. Jeong IS, Yoo JH, Seo SH, et al. Association between time (initiation and length) and oncological outcomes for the patients with colon cancer treated with adjuvant chemotherapy. Indian J Surg 2015;77:1252-7.

27. Wei XQ, Ma Y, Chen Y, et al. Laparoscopic surgery for early cervical squamous cell carcinoma and its effect on the micrometastasis of cancer cells. Medicine (Baltimore) 2018;97:e11921

28. Tsilimigras DI, Hyer JM, Paredes AZ, et al. Tumor burden dictates prognosis among patients undergoing resection of intrahepatic cholangiocarcinoma: a tool to guide post-

Cite this article as: Ren JQ, Zhang HS, Zhang LH, Zhong QG, Wu F, Wang BL, Liu SJ. Optimal adjuvant chemotherapy completion time for stage III colon cancer: a cohort study. J Gastrointest Oncol 2021;12(4):1558-1567. doi: 10.21037/jgo-21317 resection adjuvant chemotherapy? Ann Surg Oncol 2021;28:1970-8.

29. Tan K, Leong SM, Kee Z, et al. Longitudinal monitoring reveals dynamic changes in circulating tumor cells (CTCs) and CTC-associated miRNAs in response to chemotherapy in metastatic colorectal cancer patients. Cancer Lett 2018; 423:1-8.

30. Longhini F, Bruni A, Garofalo E, et al. Anesthetic strategies in oncological surgery: not only a simple sleep, but also impact on immunosuppression and cancer recurrence. Cancer Manag Res 2020;12:931-40.

31. Kadosawa T, Watabe A. The effects of surgery-induced immunosuppression and angiogenesis on tumour growth. Vet J 2015;205:175-9.

32. Pérez-García VM, Calvo GF, Bosque JJ, et al. Universal scaling laws rule explosive growth in human cancers. Nat Phys 2020;16:1232-7.

(English Language Editor: R. Scott) 